\title{
ANÁLISIS DE VIABILIDAD DE UN PROYECTO DE RECUPERACIÓN DEL PATRIMONIO CULTURAL URBANO
}

\author{
Andrea Báez \\ Universidad Austral de Chile \\ Chile \\ abaez@uach.cl \\ Luis Cesar Herrero \\ Universidad de Valladolid \\ España \\ herrero@emp.uva.es \\ Ana Bedate \\ Universidad de Valladolid \\ España \\ ana@emp.uva.es \\ Angel Sanz \\ Universidad de Valladolid \\ España \\ angel@emp.uva.es
}

\section{RESUMEN}

El plantear metodologías y realizar ensayos empíricos en torno a la valoración del patrimonio histórico y cultural, radica en que pueden servir de estimación de las preferencias de los individuos y de la sociedad, también pueden constituir un input necesario para la evaluación de políticas y proyectos concernientes al patrimonio cultural. El objetivo de este trabajo, es realizar un análisis costo-beneficio de un proyecto de recuperación del patrimonio histórico de la comuna de Valdivia en Chile. La estimación de los beneficios esperados se realiza a través de la aplicación del método de valoración contingente, concretamente para estimar el valor asignado al patrimonio cultural por parte de los ciudadanos y los turistas llegados a esta comuna. Este tipo de aplicaciones pueden resultar interesantes, por la urgencia en la recuperación de muchos de los elementos patrimoniales y la oportunidad de convertirlos en factores de desarrollo económico, o al menos de atracción turística.

Palabras clave: Economía del patrimonio histórico, valoración contingente, elección dicotómica, Circuitos turístico-culturales, análisis costo-beneficio 


\title{
FEASIBILITY ANALYSIS OF AN URBAN CULTURAL PATRIMONY RECOVERY PROJECT
}

\author{
Andrea Báez \\ Universidad Austral de Chile \\ Chile \\ abaez@uach.cl \\ Luis Cesar Herrero \\ Universidad de Valladolid \\ España \\ herrero@emp.uva.es \\ Ana Bedate \\ Universidad de Valladolid \\ España \\ ana@emp.uva.es \\ Angel Sanz \\ Universidad de Valladolid \\ España \\ angel@emp.uva.es
}

\begin{abstract}
The use of methodologies and empirical testing around the valuation of historical and cultural heritage allow the estimation of the preferences of individuals and society, they can also serve as input for the evaluation of policies and projects relating to cultural heritage. The goal of this work is to perform a cost-benefit analysis of a historical heritage restoration project in Valdivia, Chile. The estimation of the expected benefits are calculated through the implementation of a contingent evaluation method, specifically to assess the value assigned by citizens and tourists to the cultural heritage of this city. Such applications can be interesting, given the urgency to recover many of the assets and the opportunity to turn them factors of economic development, or at least as tourist attractions.
\end{abstract}

Keys word: Cultural economics heritage, contingent evaluation, dichotomous election, benefit-cost analysis. 


\section{INTRODUCCIÓN}

La economía del patrimonio histórico se configura como una rama analítica con estatus propio dentro del ámbito disciplinar de la Economía de la Cultura, debido a la particularidad de los elementos integrantes, generalmente bienes de carácter único, no reproducibles y sometidos a condiciones de sostenibilidad en el tiempo (Peacok, 1998; Hutter y Rizzo, 1997 y Herrero, 2001). Además, buena parte de estos elementos reúnen la condición de bienes públicos y proporcionan una serie de valores intangibles asociados a su contenido estético o simbólico, de manera que el mercado y los precios no resultan ser el canal más apropiado para la expresión de su valor. Ello no significa, que no exista una voluntad latente de aprecio por parte de los ciudadanos, manifestado en un deseo de recuperación y preservación de estos elementos; ni que del patrimonio cultural, entendido como una dotación de capital público, se deriven una serie de flujos de gastos y empleos asociados principalmente a la demanda turística, y que pueden contribuir al desarrollo económico de las ciudades con patrimonio cultural (Throsby, 2001). Por ello es importante plantear metodologías y realizar ensayos empíricos en torno a la valoración del patrimonio histórico y cultural, porque pueden servir, cuando menos, de estimación de las preferencias de los individuos y de la sociedad en relación a estos elementos, aunque también pueden constituir un input necesario para la evaluación de políticas culturales y proyectos de viabilidad concernientes principalmente al patrimonio histórico urbano, y por lo tanto del capital cultural de una ciudad entendido como un activo.

Esta línea de trabajo es muy importante, sobre todo en los países en desarrollo, donde existen pocos estudios que planteen la valoración de los beneficios sociales de su patrimonio cultural ${ }^{1}$, en orden a justificar, por ejemplo, la necesidad de provisión de fondos para su conservación, o bien para diseñar figuras institucionales viables que se ocupen de esta tarea. Además, el patrimonio cultural de estos países está sometido a distintos riesgos, bien por la situación de abandono en la que se encuentran y la falta de recursos dedicados a la conservación, bien por la congestión turística desmedida de determinados enclaves o la presión de otros usos alternativos de carácter especulativo, que alientan su destrucción.

De este modo, nuestra investigación corresponde a un ensayo de valoración y evaluación económica del patrimonio cultural de la comuna de Valdivia, en Chile, un conjunto histórico emblemático y un enclave cultural de primer orden en este país. Concretamente el estudio persigue dos objetivos fundamentales: primero, estimar la disposición a pagar por el patrimonio cultural, de visitantes y residentes, como forma de obtener una representación del valor económico asignado al conjunto histórico de esta comuna; y, segundo, la evaluación y el análisis de viabilidad, de un plan de recuperación y puesta en valor del patrimonio urbano a través de la creación de una hipotética fundación

1 Ver los estudios de Carson et al. (1997), Pagiola (1999), Chafla (2001) y Tuan y Navrud (2007). 
sin fines de lucro destinada a esta tarea.

Esta comunicación se estructura de la siguiente forma: la Sección 2 contiene la descripción del objeto de estudio y la metodología aplicada; la Sección 3 presenta los resultados de la estimación del valor económico del patrimonio cultural de la Comuna de Valdivia; la Sección 4 recoge la evaluación de la rentabilidad social del proyecto de recuperación de este conjunto histórico y finalmente, la Sección 5 sintetiza las principales conclusiones de la investigación.

\section{DESCRIPCIÓN DEL OBJETO DE ESTUDIO Y LA METODOLOGÍA APLICADA}

\section{Objeto de estudio y metodología}

La comuna de Valdivia forma parte de la Provincia de Valdivia en Región de los Ríos, cuenta con una población de 140.559 habitantes (92,5\% población urbana). La principal ciudad es Valdivia (Capital Regional) es un enclave urbano situado en el extremo sur de Chile, alejado de los principales centros urbanos del país y fue fundada en el año 1552 por el conquistador español Pedro de Valdivia, que escogió su ubicación estratégica entre ríos navegables cercanos a un puerto costero. El patrimonio histórico de Valdivia $^{2}$ está conformado por una serie de elementos heterogéneos y dispersos, cuyas características de identificación más significativas son su origen español, el sistema de fortificaciones del Imperio Hispano, así como la colonización alemana del siglo XIX, que dejó rasgos notables en el diseño urbano y en la identidad urbanística colectiva de la ciudad actual ${ }^{3}$.

Valdivia cuenta una serie de inmuebles declarados como "Edificios y Zonas de Conservación Histórica” que mantienen algún tipo de protección y planeamiento territorial por parte de la Municipalidad y del Ministerio de Vivienda y Urbanismo. Estos inmuebles y monumentos aparecen agrupados en ocho sectores del casco urbano (Figura 1), aunque la mayor parte de los mismos y sobre todo los más importantes y atractivos se encuentran en las zonas del centro de la ciudad, calle General Lagos e Isla Teja

2 En adelante al hacer referencia a Valdivia se está hablando de la Comuna.

3 Para mayor detalle sobre la historia y el patrimonio cultural de Valdivia puede verse Guarda (1990 y 2001) y www.monumentos.cl; 
Figura 1.- Distribución porcentual de los inmuebles en vías de conservación histórica según sector, en la comuna de Valdivia.

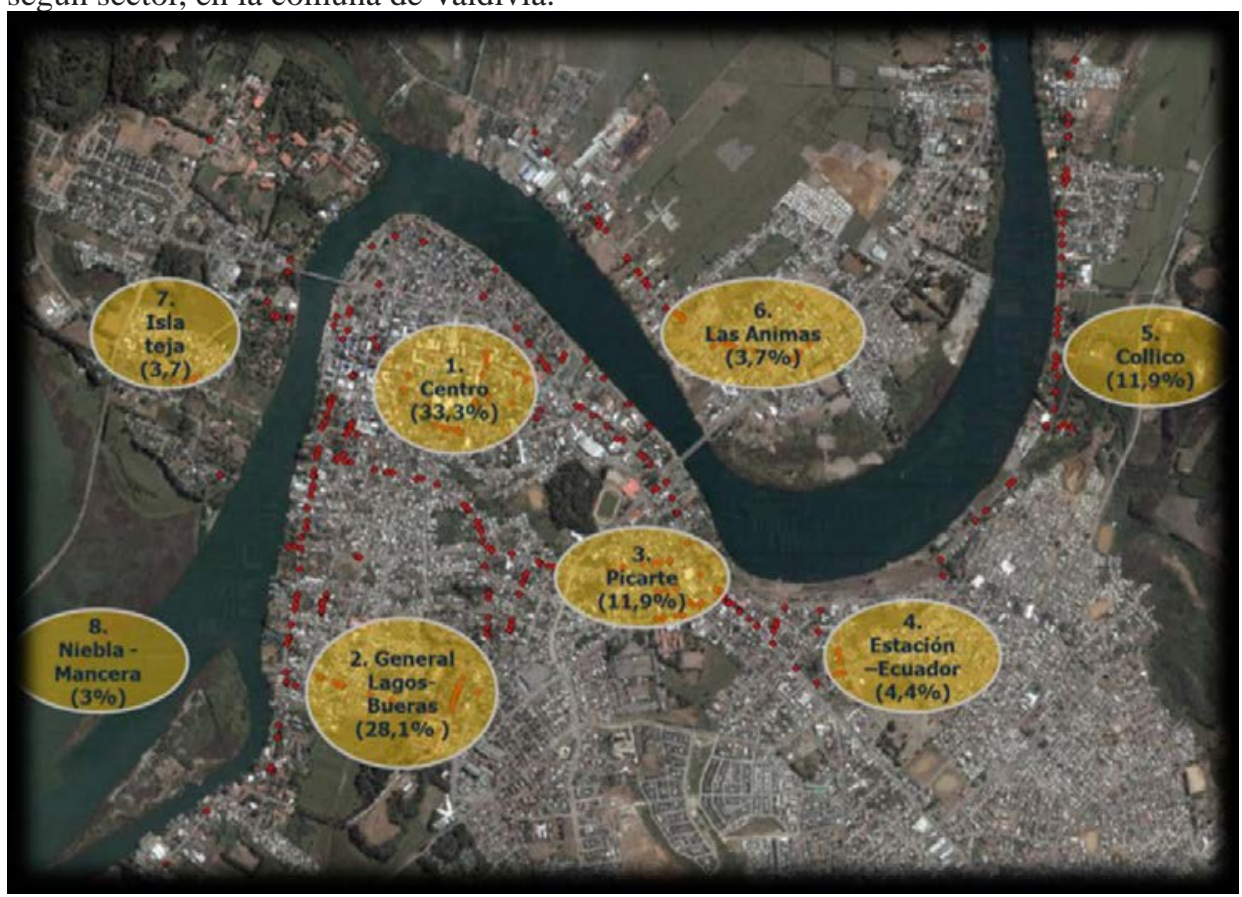

La valoración de este conjunto histórico constituye es interesante, tanto por los desafíos metodológicos que presenta, debido al carácter no acotado y disperso de los bienes objeto de estudio, como por las posibilidades de empleo de los resultados en planes de viabilidad de proyectos turísticos, o de evaluación de políticas de recuperación del patrimonio cultural. Esto es particularmente importante en el caso chileno y otros países en desarrollo, dado que las políticas de conservación del patrimonio histórico carecen de un programa de fomento con beneficios tributarios o financieros para los propietarios de bienes inmuebles de carácter histórico, de manera que muchos edificios están en proceso acelerado de destrucción, bien por la falta de recursos para su rescate, o bien porque el valor alternativo del suelo urbano fomenta su descuido con el fin de lograr otros usos, generalmente de carácter inmobiliario.

De esta forma, y por lo que se refiere al planteamiento metodológico de esta investigación, nos proponemos combinar dos tipos de aportes: la técnica de valoración contingente para el estudio de la asignación de valor, y el análisis costo beneficio para la evaluación de política elegida. En la primera etapa, la técnica de valoración contingente nos entrega las estimaciones de la Disposición a Pagar (DAP) de distintos individuos 
por el patrimonio cultural de Valdivia a través de dos aproximaciones: por un lado, en forma del valor proporcionado por los turistas llegados a Valdivia y, por otro, a través del valor indicado por los residentes de la comuna. Ambos resultados se utilizaran como insumos en el análisis de costo-beneficio (ACB), concretamente como estimación de los beneficios sociales que procura el patrimonio cultural de la comuna. Al comparar estos beneficios con los costos en que se debe incurrir con la implementación de una fundación dedicada al rescate y promoción del patrimonio cultural urbano, podemos determinar si el proyecto es rentable socialmente o no, a través de distintos indicadores de viabilidad. Esta tarea comporta de forma adicional un esfuerzo previo de diseño institucional de la política cultural en cuestión

\section{VALORACIÓN CONTINGENTE DEL PATRIMONIO HISTORICO DE LA COMUNA DE VALDIVIA (CHILE)}

\section{Especificación del modelo}

El método de valoración contingente (Mitchell y Carson, 1989; Bateman y Willis, 1999) es un método directo de estimación de preferencias declaradas por un bien que no se transa en el mercado, donde los individuos son preguntados por su disposición a pagar (DAP) por los beneficios recibidos en un cambio en la provisión de este bien. En términos teóricos, el método de valoración contingente está basado en la economía del bienestar, porque asume que los valores de la DAP están directamente relacionados con la función de preferencias de los individuos.

De esta forma, el marco teórico elemental de los estudios de valoración contingente considera una función de utilidad de un individuo, u, que depende de bienes de mercado, $\mathrm{x}$, y de un nivel de provisión de un bien público, q. Los individuos minimizan los gastos px sujetos a un valor dado de la función de utilidad $u=u^{*}(x, q)$, de manera que la función de gasto se expresa como $e=e\left(p, q 0, u^{*}\right)$, donde $q_{0}$ es el valor corriente del bien público. A partir de aquí, la DAP de los individuos que responden por un cambio desde $q_{0}$ a $q_{1}$ se define como:

$\operatorname{DAP}(q)=e\left(p, q 0, u^{*}\right)-e(p, q 1, u(1)$

La obtención de la DAP puede realizarse de muy diversas maneras, que van desde las preguntas sencillas de carácter abierto, a juegos de subastas más o menos complicados (Bishop y Haberlein, 1979; Hanemann, 1984; Carson et al., 1997); todo ello con el objetivo de encontrar el verdadero valor de la DAP, que sólo conoce de manera certera el encuestado, pero no el investigador. Una vez capturados estos valores declarados, intentando minimizar los distintos sesgos que inevitablemente aparecen en un procedimiento de encuestación de estas características, la DAP puede estimarse a través de diversos métodos econométricos. Los modelos de regresión de carácter paramétrico 
han sido profusamente utilizados en este sentido (Cameron y James, 1987; Hanemann et al., 1991 y Cameron y Quiggin, 1994) en la idea de que la DAP está estrechamente conectada con las características demográficas y socioeconómicas de los individuos, así como con las experiencias previas en el consumo del bien en cuestión y la disponibilidad de sustitutos

Sin embargo, la excesiva rigidez que involucran formas funcionales específicas en la explicación del comportamiento del consumidor, ha incentivado la estimación de la DAP por medio de métodos no paramétricos (Cucccia y Signorelo, 2002; Sanz et al., 2003, Saz y Montagud, 2005, Bedate et al., 2006). La idea básica reside en que a priori no existe ninguna razón que justifique que los gustos de los consumidores sigan una distribución particular, y las estimaciones no paramétricas maximizan la flexibilidad y minimizan, aunque no eliminan, los supuestos necesarios para estimar la función de supervivencia.

Los modelos no paramétricos aplicados en valoración contingente son análogos a aquellos que involucran la estimación de la función de supervivencia, en aplicaciones en las cuales interesa estimar la probabilidad de que el tiempo de ocurrencia de un fenómeno sea al menos t (Greene, 1998). El método consiste en la estimación de las probabilidades a aceptar los pagos iniciales a través de algún algoritmo o procedimiento iterativo que permita encontrar la solución óptima y convergente. La variable tiempo comúnmente utilizada en otra aplicaciones se sustituye por la máxima disposición a pagar subjetiva del individuo, y el suceso a predecir corresponde a la respuesta favorable al escenario de valoración propuesto.

En esta investigación se utiliza el algoritmo no paramétrico propuesto por An y Ayala (1996), el cual consiste en una versión mejorada del método de Turnbull, pues con él se puede tratar datos agrupados arbitrariamente, que es un esquema común dentro de la valoración de bienes públicos.

En el ámbito de la valoración de bienes culturales existen también opiniones divergentes ${ }^{4}$, entre quienes ven en estas aplicaciones un mal menor asumible por el momento como expresión monetaria de las preferencias de los individuos; y quienes se inquietan porque estas técnicas nunca pueden llegar a capturar el valor cultural de los bienes y las obras de arte, puesto que se trata de un concepto poliédrico que no se expresa sólo en forma de dinero. Aun siendo conscientes de estas críticas, hemos de reconocer el gran predicamento actual de los ejercicios de valoración contingente en el campo de la valoración de los bienes públicos y, particularmente, de elementos del patrimonio histórico. La eficiencia de la técnica y la utilidad de sus resultados dependen, en gran medida, de la escrupulosidad en el procedimiento; pero cuando menos ofrecen 4 Ver el número monográfico del Journal of Cultural Economics (vol. 27, núm. 3-4, noviembre 2003) dedicado especialmente al método de valoración contingente en la economía de la cultura. 
una jerarquía consistente de las preferencias individuales, y también de las preferencias sociales si, por ejemplo, los esquemas de financiamiento se basan en aportes voluntarios para la provisión de un bien público (Cuccia, 2003). Desde este punto de vista, el método de valoración contingente puede ser una herramienta muy útil para los responsables públicos del patrimonio cultural, ya que sus resultados pueden servir de pauta coherente para los criterios de provisión de fondos o para la evaluación de acciones normativas.

\section{Valor del patrimonio histórico urbano asignado por los turistas}

La población objetivo para el primer estudio de valoración está constituida por los turistas mayores de 18 años que visitaron a Valdivia en los meses del verano del año 2009. El tamaño de la muestra correspondió a un error del $3 \%$ con un $95 \%$ de confianza; y fueron entrevistados un total de 1032 visitantes en forma aleatoria. Las entrevistas fueron personales por medio de un grupo de reducido de agentes previamente entrenados; y finalmente se tomaron 922 respuestas válidas, a través del filtro de voluntad positiva de participación en el mercado hipotético planteado.

El cuestionario fue estructurado en tres partes: la primera gira en torno a las actividades desarrolladas por el turista entrevistado (nacionalidad, medio de transporte, lugar de hospedaje, número de acompañantes, gasto aproximado, etc.); la segunda parte se centra en la valoración monetaria del objeto de estudio, mediante la exposición de la pregunta de valoración contingente y el vehículo de pago; por último, la tercera parte trata de recabar las características socioeconómicas del entrevistado incluyendo sexo, edad, estudios realizados, renta, etc.

Puesto que se trata de capturar el valor asignado por los turistas al patrimonio histórico de la Comuna de Valdivia, el principal problema para acotar el objeto de estudio surge de la dispersión en que se encuentran los bienes patrimoniales de esta ciudad, que no están circunscritos por ninguna muralla (como se observa en la Figura 2), o en un centro histórico, como ocurre normalmente en países europeos. De esta forma se adoptó una estrategia metodológica para superar este obstáculo, proponiendo tres "circuitos guiados", de manera tal que el bien a valorar esté claramente definido y sea lo más real posible Estos circuitos se titularon específicamente en la encuesta como Circuito Centro-Oeste (color amarillo); Circuito Centro Oriente (color verde) y Circuito centro sur (color rojo) ellos recorren los principales hitos culturales y patrimoniales de la comuna de Valdivia (Figura 2), la diferencia entre los circuitos contempla, extensión, contenido de patrimonio histórico y natural en mayor o menor proporción, posibilidad de visitar museos, etc.

5 Un planteamiento metodológico similar puede verse en Santagata y Signorello (2000) o Baez (2007), sim embargo la diferencia es que los circuitos en estas investigaciones son peatonales. 
Figura 2. Patrimonio cultural de Valdivia: propuesta de Circuitos turísticos - históricos

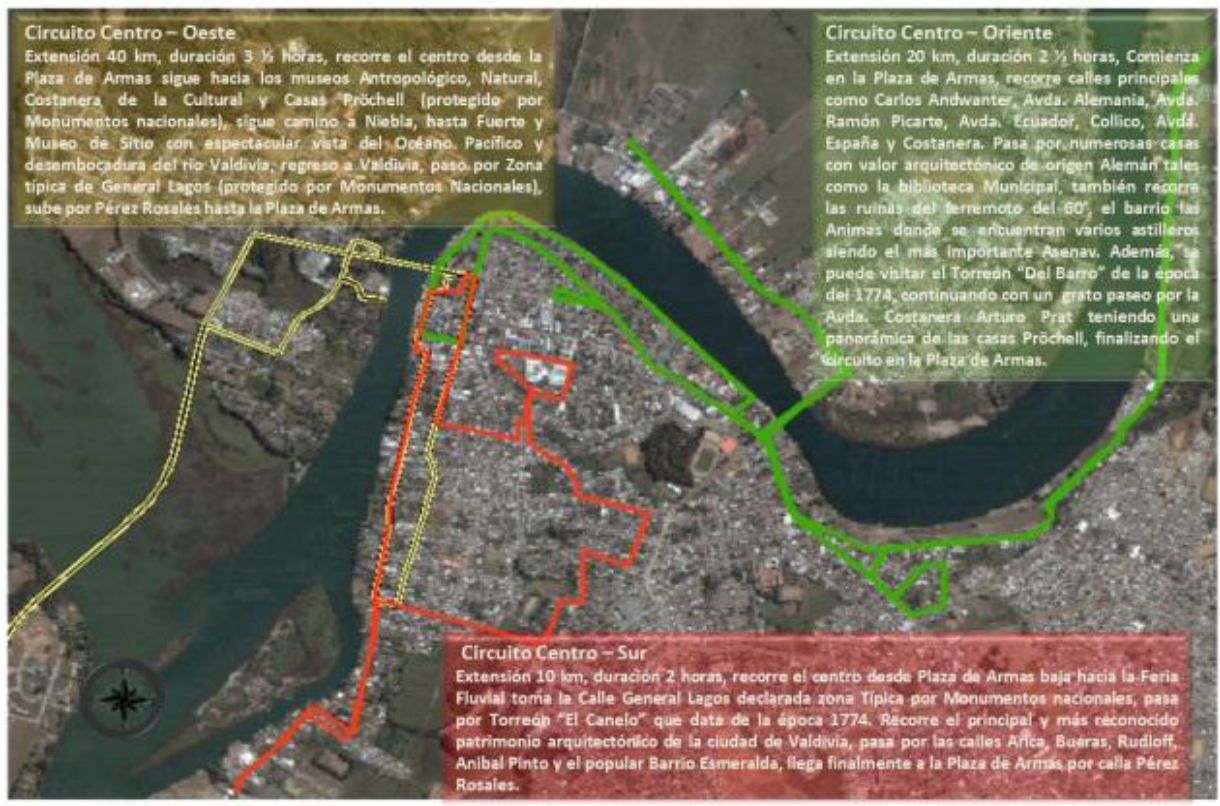


Figura 2. Patrimonio cultural de Valdivia: propuesta de Circuitos turísticos - históricos

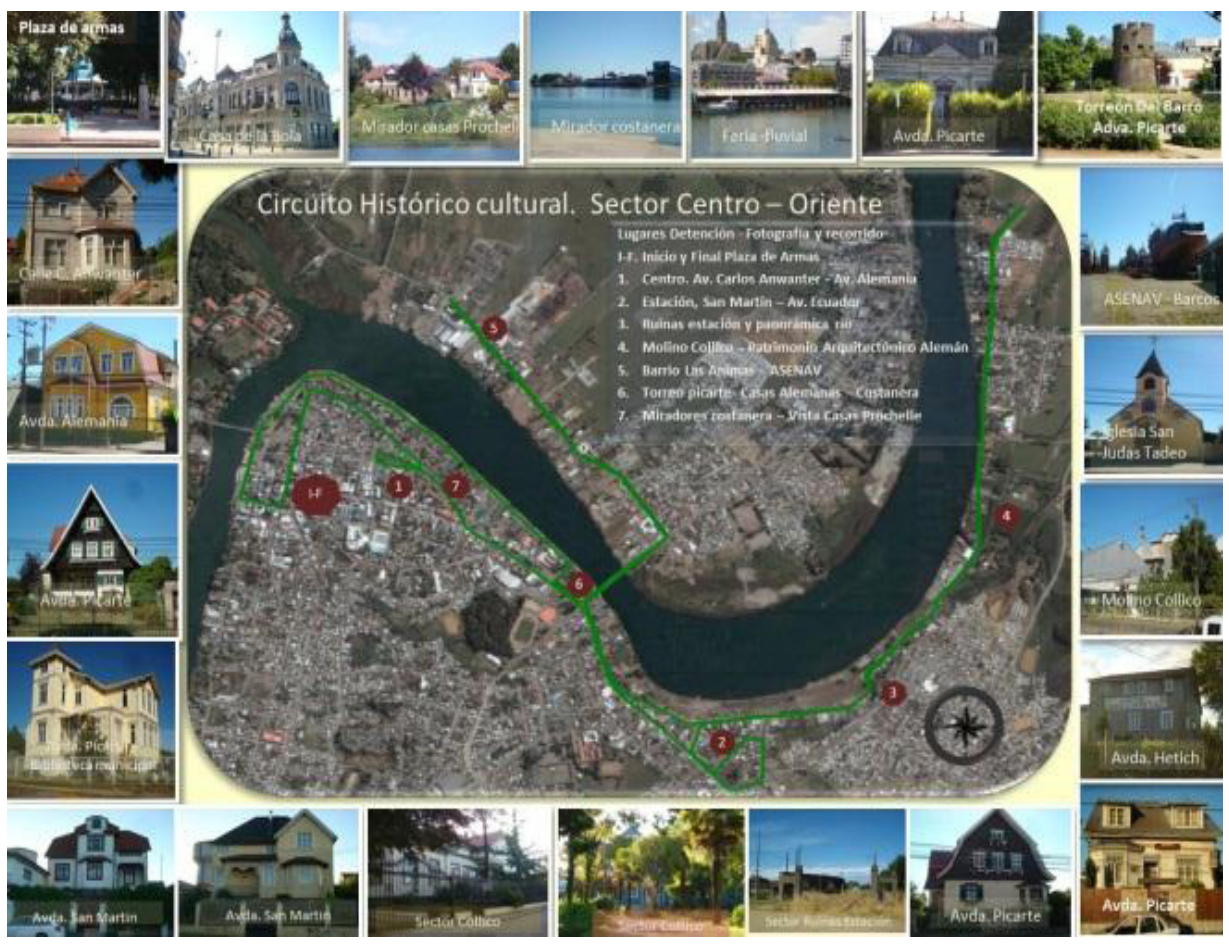


Figura 2. Patrimonio cultural de Valdivia: propuesta de Circuitos turísticos - históricos

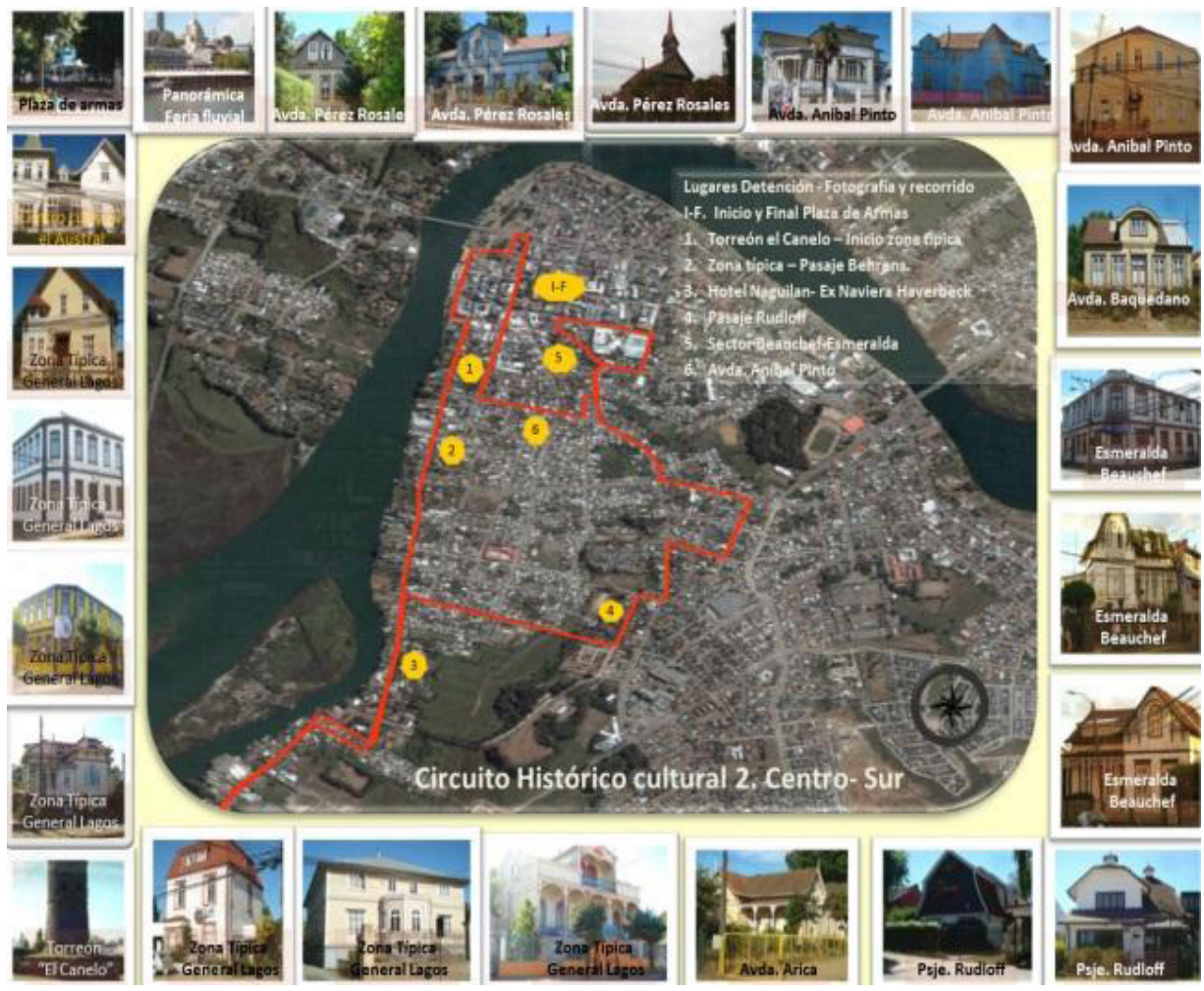


Figura 2. Patrimonio cultural de Valdivia: propuesta de Circuitos turísticos - históricos

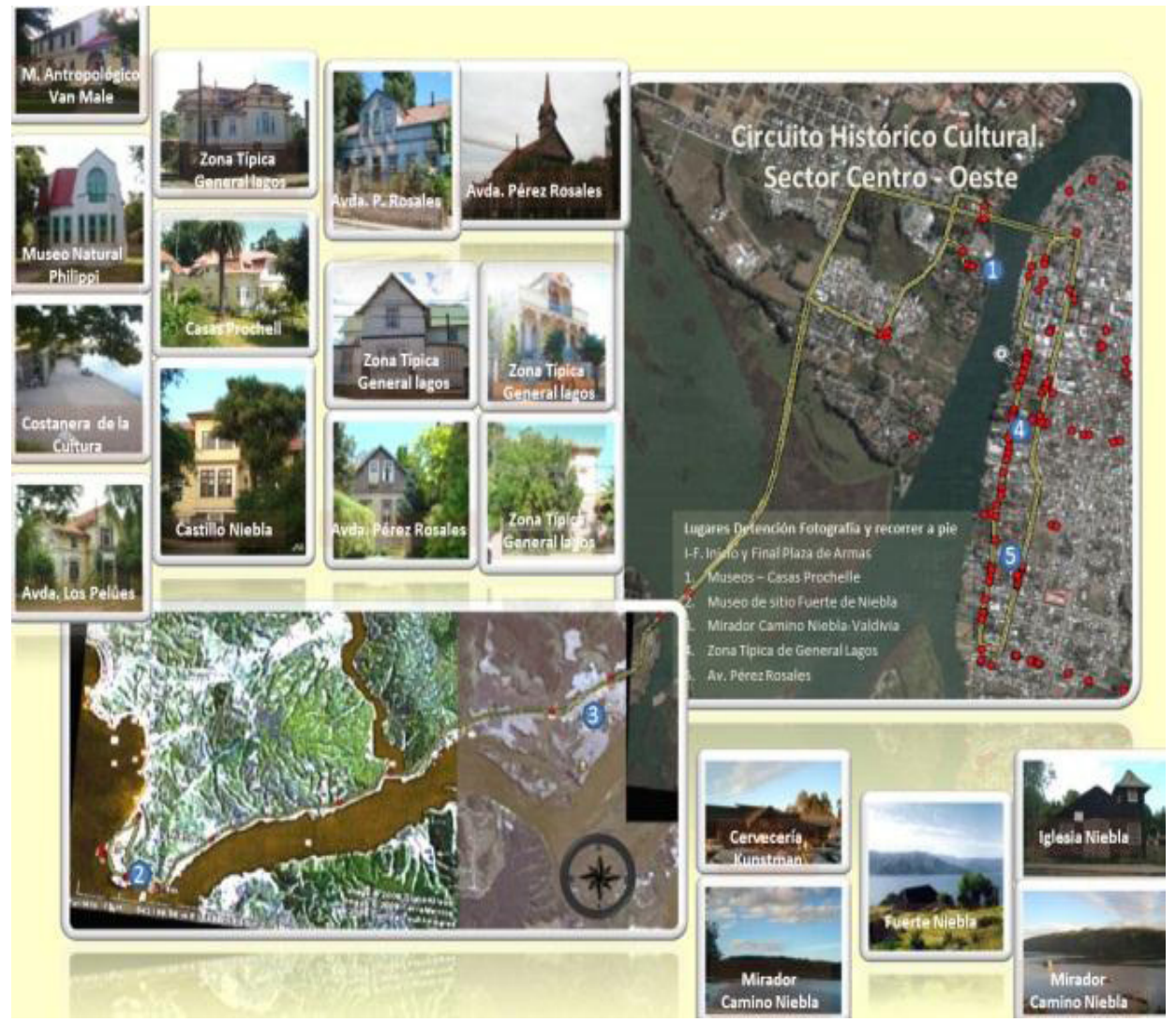


El vehículo de pago de la pregunta de valoración fue un ticket de acceso. Como método de subasta, se empleó el método dicotómico doble con un vector de seis precios de salida, escogido de acuerdo a la distribución empírica de las respuestas obtenidas en un ejercicio de pre-encuesta con pregunta abierta, y posteriormente ajustados utilizando los datos recogidos en la encuesta piloto. El Cuadro 1 presenta los precios del boleto en pesos chilenos y dólares ${ }^{6}$ asignados en forma aleatoria a los individuos de la muestra. Si la respuesta del individuo al primer precio es afirmativa, se presenta un precio mayor, como vemos en la segunda fila del cuadro; y en caso contrario, un precio inferior, que aparece en la tercera fila?

Cuadro 1.- Vector de precios en pesos chilenos (dólares) presentado a los entrevistados para el formato de doble acotación.

\begin{tabular}{|l|c|c|c|l|l|l|}
\hline Primer Precio & $\begin{array}{c}\mathbf{2 . 0 0 0} \\
\mathbf{( 3 , 3 )}\end{array}$ & $\begin{array}{c}\mathbf{3 . 5 0 0} \\
\mathbf{( 5 , 8 )}\end{array}$ & $\begin{array}{c}\mathbf{5 . 0 0 0} \\
\mathbf{( 8 , 3 )}\end{array}$ & $\begin{array}{c}\mathbf{7 . 0 0 0} \\
(\mathbf{1 1 , 7}\end{array}$ & $\begin{array}{l}\mathbf{1 0 . 0 0 0} \\
\mathbf{( 1 6 , 7 )}\end{array}$ & $\begin{array}{l}\mathbf{1 5 . 0 0 0} \\
\mathbf{( 2 5})\end{array}$ \\
\hline $\begin{array}{l}\text { Segundo precio/Si al } \\
\text { primer precio }\end{array}$ & $\begin{array}{c}3.500 \\
(5,8)\end{array}$ & $\begin{array}{c}5.000 \\
(8,3)\end{array}$ & $\begin{array}{c}7.000 \\
(11,7)\end{array}$ & $\begin{array}{c}10.000 \\
(16,7)\end{array}$ & $\begin{array}{l}15.000 \\
(25)\end{array}$ & $\begin{array}{c}25.000 \\
(41,7)\end{array}$ \\
\hline $\begin{array}{l}\text { Segundo precio/No } \\
\text { al primer precio }\end{array}$ & $\begin{array}{c}1.000 \\
(1,7)\end{array}$ & $\begin{array}{l}2.000 \\
(3,3)\end{array}$ & $\begin{array}{c}3.500 \\
(5,8)\end{array}$ & $\begin{array}{l}5.000 \\
(8,3)\end{array}$ & $\begin{array}{l}7.000 \\
(11,7)\end{array}$ & $\begin{array}{c}10.000 \\
(16,7)\end{array}$ \\
\hline
\end{tabular}

Fuente: Elaboración propia

Los resultados obtenidos después de la aplicación del algoritmo de An y Ayala nos indican que al calcular el excedente del consumidor sobre la curva de supervivencia empírica, tomada como curva de demanda de los turistas llegados a Valdivia, podemos decir que la DAP media de cada uno de los individuos, general sin importar el circuito fue de 8.328 pesos chilenos (13,9US\$). Este valor representa la disposición media a pagar de cada uno de los turistas por acceder a alguno de los tres tour guiados propuestos, y tomado como estimación del valor de uso directo del patrimonio histórico de la comuna de Valdivia. Debemos mencionar que al realizar la estimación de la DAP entre los tres tour, se encontró que ésta es levemente mayor en el circuito Centro-sur (rojo), seguido de circuito Centro-oriente (verde) y el más bajo fue el circuito Centro-oeste (amarillo). En todo caso, y a efectos operativos de tomar un precio único no discriminatorio, se optó por considerar el resultado de la DAP media del conjunto de los turistas.

\section{Valor del patrimonio histórico urbano asignado por los residentes}

El estudio realizado para estimar el valor asignado por los residentes, se efectuó mediante una encuesta cara a cara a los habitantes, mayores de 18 años, durante el

6 El tipo de cambio en 2009 era de 1 Euro a 750 pesos y las relaciones con el dólar son 1 US\$, 600 pesos.

7 Estos vectores se escogieron de tal forma que la probabilidad de responder afirmativamente al máximo precio ofrecido se aproximase a cero, mientras la probabilidad de responder afirmativamente al mínimo precio se acercase a la unidad. 
periodo comprendido entre los meses de junio a octubre de 2008. El tamaño de la muestra correspondió a un error del 2,95\%, con un $95 \%$ de confianza. Finalmente, para lograr la muestra de resultados válidos de 816 encuestas que aceptaron el mercado contingente.

El cuestionario fue estructurado, al igual que en la encuesta anterior, en tres partes: la primera gira en torno al consumo cultural por parte del entrevistado, la segunda se centra en la valoración monetaria del objeto de estudio; y la tercera recoge los aspectos socioeconómicos de los individuos. En este caso, el objeto de estudio no se circunscribe a los circuitos propuestos, sino que comprende el conjunto patrimonial urbano global de Valdivia. Como vehículo de pago se propuso una cuota de donación anual a una Fundación sin fines de lucro encargada de la recuperación y mantenimiento de este patrimonio. Con este planteamiento queremos acercarnos al concepto de valor de existencia y valor de legado expresado por los ciudadanos de Valdivia respecto de su patrimonio, y se corresponde con una de las formas más habituales de financiación de los bienes públicos locales a través de contribuciones financieras, en este caso de carácter voluntario. Para el vector de ofertas iniciales se recurrió al utilizado en el estudio de Báez (2007), que es un vector de precios más amplio en comparación con la encuesta realizada a los turista, (ver cuadro 2), pues consideramos que estas cifras son adecuadas para la realidad de la situación socioeconómica de los residentes de la comuna, en un ejercicio de valoración absolutamente novedoso en el ámbito cultural de Chile. El formato de licitación fue también el método dicotómico de doble acotación.

Cuadro 2.- Vector de precios en pesos chilenos (dólares) presentado a los entrevistados para el formato de doble acotación.

\begin{tabular}{|l|l|l|l|l|l|l|l|l|}
\hline Primer Precio & $\begin{array}{l}\mathbf{2 . 0 0 0} \\
\mathbf{( 3 , 3 )}\end{array}$ & $\begin{array}{l}\mathbf{3 . 0 0 0} \\
\mathbf{( 5 , 0 )}\end{array}$ & $\begin{array}{l}\mathbf{4 . 3 0 0} \\
\mathbf{( 7 , 2 )}\end{array}$ & $\begin{array}{l}\mathbf{5 . 7 0 0} \\
\mathbf{( 9 , 5 )}\end{array}$ & $\begin{array}{l}\mathbf{7 . 5 0 0} \\
\mathbf{( 1 2 , 5 )}\end{array}$ & $\begin{array}{l}\mathbf{9 . 5 0 0} \\
\mathbf{( 1 5 , 8 )}\end{array}$ & $\begin{array}{l}\mathbf{1 2 . 0 0 0} \\
\mathbf{( 2 0 , 0 )}\end{array}$ & $\begin{array}{l}\mathbf{1 5 . 0 0 0} \\
\mathbf{( 2 5 , 0 )}\end{array}$ \\
\hline $\begin{array}{l}\text { Segundo } \\
\text { precio/ Si al } \\
\text { primer precio }\end{array}$ & 2.500 & 3.500 & 5.000 & 6.500 & 8.500 & 10.500 & 13.500 & 20.000 \\
$(4,2)$ & $(5,8)$ & $(8,3)$ & $(10,8)$ & $(14,2)$ & $(17,5)$ & $(22,5)$ & $(33,3)$ \\
\hline $\begin{array}{l}\text { Segundo } \\
\text { precio/No al } \\
\text { primer precio }\end{array}$ & 1.500 & 2.500 & 3.500 & 5.000 & 6.500 & 8.500 & 10.500 & 13.500 \\
$(2,5)$ & $(4,2)$ & $(5,8)$ & $(8,3)$ & $(10,8)$ & $(14,2)$ & $(17,5)$ & $(22,5)$ \\
\hline
\end{tabular}

Fuente: Elaboración propia

Los resultados de la estimación no paramétrica de la DAP de los ciudadanos de Valdivia respecto de su patrimonio histórico utilizando el algoritmo de An y Ayala, considerando el valor del excedente del consumidor de la curva de supervivencia empírica, podemos calcular que la DAP media es 9.207 pesos chilenos (15,3US\$).

Las cifras estimadas son la comprobación de una voluntad de pago positiva, tanto de los visitantes a la ciudad como de los propios residentes de la misma y representan un 
resultado apreciable como expresión de las preferencias declaradas por estos individuos y, por tanto, del valor del patrimonio cultural de la comuna. Así mismo pueden constituir un dato importante para la evaluación de proyectos de viabilidad cultural asociadas al patrimonio histórico, como pretendemos demostrar seguidamente.

\section{EVALUACIÓN DE LA RENTABILIDAD SOCIAL DE UN PROYECTO DE RECUPERACIÓN DEL PATRIMONIO CULTURAL URBANO}

\section{Antecedentes y diseño institucional del proyecto}

En la actualidad las funciones de rescatar y conservar este patrimonio carecen de un organismo o entidad local que fomente o se haga cargo de esta vital tarea, en tanto que sólo existen normas de protección genérica para determinados inmuebles que cuentan con una tipificación como monumento nacional. Es por este motivo que nos planteamos en esta investigación la idea de implementar un proyecto cultural tendente a crear una Fundación sin fines de lucro cuya función primordial sería gestionar un plan de recuperación urbana y cultural para el conjunto histórico de Valdivia.

En consecuencia, este proyecto contiene, en primer lugar, un esfuerzo de diseño institucional y determinación de para el nuevo organismo. De este modo, las actividades fundamentales a desarrollar por esta Fundación giran en torno a tres líneas: una principal de recuperación y mantenimiento del patrimonio histórico urbano, otra de gestión de productos turísticos relacionados, y una tercera referida a las tareas de difusión de sus actividades y promoción del patrimonio cultural valdiviano. Como esquema de gestión se plantea uno extremadamente simple: por un lado, los costos consisten en las actividades de rescate y restauración del patrimonio, así como los de puesta en marcha y desarrollo de la Fundación; y por otro lado, la estimación de ingresos provienen de cuatro vías que son, los ingresos de algunos productos turísticos (circuitos guiados), las contribuciones voluntarias de ciudadanos y agentes privados, el aporte de los propietarios de los elementos del patrimonio urbano a restaurar, y finalmente una partida abierta de ingresos externos por mecenazgo de otros agentes.

Se trata, por tanto, de la formulación de una política cultural muy sencilla y operativa, de carácter aplicado porque se nutre de los resultados de la propia investigación, como son las estimaciones del valor económico asignado al patrimonio cultural de la Comuna de Valdivia, tanto por los turistas como por los propios residentes. Estos valores serán considerados como una forma de rentabilidad del patrimonio cultural y, por tanto, partidas de ingresos del proyecto planteado.

Para la evaluación de esta intervención cultural y su propuesta institucional se 
emplea, quizás, una de las técnicas de valoración de proyectos más conocidas y utilizadas, como es el análisis costo-beneficio (Mischan,1971 y Pearce y Nash, 1981 entre los más recientes encontramos a; Boardman et al., 2001 y Sen, 2000), donde la particularidad, en nuestro caso, es la consideración del rendimiento social de algunos bienes que no se expresan bien a través del mercado, como el valor del patrimonio histórico. Los aspectos técnicos principales a tener en cuenta en un análisis de este tipo tienen relación, primero, con los proyectos alternativos al planteado, que para nuestro estudio usaremos el criterio de status quo, es decir, que se analizará la viabilidad del plan desde la perspectiva de que no hay ninguna otra política en ejecución con el mismo propósito. A continuación han de cuantificarse e identificarse, tanto los beneficios, como los costes del proyecto, así como estimar un período razonable de ejecución, que en nuestra aplicación se estima en un intervalo de diez años. Otro de los puntos fuertes del análisis es la determinación de la tasa social de descuento, que se estipulará inicialmente en un $10 \%{ }^{8} 9$. La viabilidad del plan será juzgada a través de ratios financieros comunes y comprensibles como son el cálculo del valor actual neto (VAN) y la tasa interna de retorno (TIR).

\section{Identificación de los costos}

Los principales costos considerados en el análisis coste beneficio de este proyecto cultural están divididos en dos partes, una relacionada directamente con la implementación de la Fundación propiamente dicha y la otra con las actividades de recuperación del patrimonio histórico de la comuna, y particularmente con el hermoseamiento de los tour antes mencionados. A su vez, el primer grupo de costos ha sido clasificado en tres partes: costos legales de creación de la institución, costos de infraestructura y dotación de equipamientos, y finalmente, los costos de personal.

En otro orden de cosas y en relación al grupo de costes de recuperación y puesta en valor del patrimonio cultural urbano de Valdivia, éstos están conformados por: restauración y mantención de las casas patrimoniales, señalética de los circuitos y actividades de difusión y promoción de las labores de la Fundación.

\section{Identificación y cuantificación de beneficios}

Una de las dificultades del análisis costo beneficio es estimar los beneficios cuando el bien no se transa en el mercado, situación que concuerda con nuestro objeto de

\footnotetext{
8 Tanto el periodo de análisis como las tasas de descuento utilizadas, están basadas en propuestas de distintos organismos y proyectos públicos de carácter social (Comisión Europea, 1997 y 2003; ONUDI, 1993; CEPAL, 2005). No existe ningún referente comparativo de este tipo de estudios en el sector cultural y en Chile. No obstante, podemos indicar, a modo de referencia, que la tasa nominal de interés a largo plazo en Chile según el Banco Central es de un 4,9\%, por lo que cómo mínimo la tasa social de descuento debería ser superior a esta cifra. Si bien la elección de la tasa de descuento es una cuestión clave en el ACB (Rus, 2004), al realizar posteriormente un análisis de sensibilidad de los resultados frente a distintas cifras alternativas, se justifica la opción finalmente elegida.
} 
estudio, el patrimonio histórico cultural de la comuna de Valdivia, sobre el que, además, no existe un plan de aprovechamiento turístico específico. De esta manera, nuestro aporte metodológico consiste en utilizar las estimaciones de la DAP, obtenidas por el método de valoración contingente, como representativas de la rentabilidad social que posee el conjunto patrimonial y, por tanto, como insumo en el análisis costo-beneficio. Concretamente, la estimación del valor de uso directo por parte de los turistas a través del ticket de entrada a alguno de los tours guiado, será considerado como un ingreso directo para el proyecto cultural, proveniente de un producto específicamente mercantil como es la explotación del circuito turístico. Por otro lado, la estimación del valor de uso pasivo de los ciudadanos de Valdivia en forma de cuota de donación voluntaria, será contemplada también como posible contribución financiera a las actividades de la Fundación sin fines de lucro. Finalmente hay que considerar como ingreso directo el propio plan de cofinanciación por parte de los dueños de los inmuebles incluidos en la restauración ${ }^{9}$ A continuación presentamos la forma de obtención y agregación de los distintos beneficios considerados en el proyecto.

Por lo que se refiere al valor de uso directo, tomamos como estimación la DAP expresada por los turistas llegados a Valdivia $(\$ 8.327 \text { pesos })^{10}$. Considerando un promedio de 90 turistas que tomen el tour diariamente en el periodo estival (100 días), podemos calcular el beneficio social agregado por año de la forma siguiente:

$$
\text { Dap agregado }=90 * 100 * 8.327 / 3=24,981 \text { millones }
$$

Por lo que se refiere al valor de uso pasivo, tomamos en cuenta la estimación de las contribuciones voluntarias de los ciudadanos de Valdivia para el mantenimiento del patrimonio cultural. En este caso partimos de la muestra realizada en la aplicación empírica en nueve sectores de la comuna, tomando en cuenta que el $\mathrm{n}^{\circ}$ de hogares de la comuna (proyectados al 2009) alcanzan las 47 mil viviendas. Entonces, considerando que el porcentaje de participación voluntaria en este sufragio sobre el patrimonio cultural de la ciudad fue del 74,2\% y la estimación de la DAP fue de 9.208 pesos, llegamos a que el beneficio social estimado por año para el conjunto de residentes es de:

$$
\text { Dap agregado }=47 \mathrm{mil} * 0.742 * 9.208 / 3=107,040 \text { millones }
$$

Por último, se considera que los dueños de casas patrimoniales deben realizar un aporte mínimo de al menos el 10\% del coste de recuperación de su vivienda, de modo 9 La posibilidad de ingresos extra por mecenazgo privado de otras instituciones no se consideran en este momento, puesto que se trataría de una partida discrecional, sujeta a la capacidad de gestión de la Fundación y la consolidación de sus actividades. Puesto que se trata de ingresos adicionales al proyecto, ello nos reafirma en que las estimaciones de este plan de viabilidad son prudentes y no sobrevaloradas.

10 Para agregar los beneficios tanto de turistas como de residentes se incorporó el sesgo hipotético y como lo establecen Harrison y Rutström (2005), Harrison (2006), NOAA (1994) y Bedate et al., (2009) se decidió dividir la DAP estimada por tres, para hacer más conservadora y realistas el cálculos de los beneficios. 
que se sientan participes de las reformas de su bien raíz, pero también conscientes de contribuir al objetivo global de recuperación del conjunto histórico de la ciudad. Esta cifra se obtiene calculando el $10 \%$ del apartado de restauración de fachadas, y debe considerarse como beneficio o ingreso específico del proyecto.

No puede olvidarse que un proyecto de este tipo genera externalidades positivas que redundan en beneficio de su propósito fundacional, como pueden ser el mayor atractivo turístico a medio plazo de un conjunto patrimonial restaurado, que atraería más turistas; o la inercia en el proceso de concienciación colectiva de los ciudadanos de Valdivia en función del éxito en las actividades de la Fundación, que captaría más donaciones. Sin embargo estos efectos no son objetivables de la misma forma en que lo hemos hecho con la estimación de los beneficios sociales del patrimonio cultural urbano, por lo que no han sido considerados en el análisis. En consecuencia, pensando que los ingresos por concepto de la venta de tour peatonal guiado (turistas) y contribución voluntaria de los residentes se computan sólo a partir del segundo año, ya que se deja el primer período para la conformación de la Fundación y la difusión inicial de sus actividades.

\section{Determinación viabilidad del proyecto}

La evaluación de la viabilidad social del proyecto de recuperación del patrimonio histórico urbano se realizó en base al VAN, que nos entrega un valor de la rentabilidad social (positiva o negativa) en la moneda de referencia y la TIR, que nos indica la tasa que iguala el VAN a cero.

Como se indicó al inicio de esta Sección, se va a usar inicialmente una tasa de descuento del 10\% para estimar el VAN del proyecto global, y concretamente sobre un flujo de costes y beneficios que, además, se encuentran adaptados al IPC de Chile ${ }^{11}$, tal y como se refleja en el Cuadro 3. De esta forma, el proyecto aparece como claramente rentable, ya que el valor actual neto del conjunto presenta una cantidad positiva de 8,939 millones de pesos.

11 La estimación del IPC se obtuvo calculando un promedio geométrico para el IPC de los últimos 10 años en Chile, arrojando un valor estimado de 3,4\%. Las series del IPC se pueden ver en Banco Central de Chile (2009). 
Cuadro 3.- Distribución de flujos para el cálculo del VAN (pesos), con tasa 10\%

\begin{tabular}{|l|l|l|l|l|l|}
\hline Año & $\begin{array}{l}\text { Coste } \\
\text { Adaptados IPC }\end{array}$ & $\begin{array}{l}\text { Beneficios } \\
\text { Adaptados IPC }\end{array}$ & St & $(1+\mathrm{i}) \mathrm{t}$ & $\begin{array}{l}\text { Saldo neto } \\
\text { anual }\end{array}$ \\
\hline 1 & 128,7659 & 8,109 & $-120,657$ & 1,000 & $-120,657$ \\
\hline 2 & 134,724 & 142,730 & 8,007 & 1,100 & 7,279 \\
\hline 3 & 136,042 & 146,600 & 10,558 & 1,210 & 8,726 \\
\hline 4 & 146,490 & 151,585 & 5,095 & 1,331 & 3,828 \\
\hline 5 & 106,376 & 151,387 & 45,010 & 1,464 & 30,743 \\
\hline 6 & 114,774 & 156,534 & 41,760 & 1,611 & 25,930 \\
\hline 7 & 126,186 & 161,856 & 35,671 & 1,772 & 20,135 \\
\hline 8 & 136,128 & 167,148 & 31,020 & 1,949 & 15,918 \\
\hline 9 & 148,785 & 172,831 & 24,046 & 2,144 & 11,218 \\
\hline 10 & 164,983 & 178,707 & 13,724 & 2,358 & 5,820 \\
\hline & & & & VAN & $\mathbf{8 , 9 3 9}$ \\
\hline
\end{tabular}

Por otra parte, la tasa interna de retorno o tasa a la que se iguala el valor presente de los beneficios y costes del proyecto, se calcula sobre los datos del Cuadro 9 y arroja un valor de 11,6\%. Este es un dato significativo, pues representa la tasa de rendimiento implícito del proyecto, con independencia de cualquier tipo de descuento. De este modo, un criterio de evaluación podría ser desechar los planes que tuviesen una TIR por debajo de determinado valor, o bien, aceptar todos aquellos en los que ésta exceda a la tasa social de descuento. En cualquier caso, la TIR de nuestro proyecto objeto de evaluación es considerablemente elevada como para considerar que el plan es rentable en términos sociales.

La incertidumbre de las previsiones que se desprenden del análisis coste beneficio tiene su origen en diversas causas. En una segunda etapa de la presente investigación se realizará un análisis de sensibilidad cuyo objetivo es la selección de las variables y parámetros "críticos" del modelo, es decir, aquellos cuyas variaciones positivas o negativas, frente al valor utilizado como estimación óptima en la hipótesis de referencia, tienen efecto más pronunciado sobre la TIR, el VAN. 


\section{CONCLUSIONES}

El patrimonio histórico de la comuna de Valdivia está conformado por una serie de elementos heterogéneos y dispersos, cuyas características de identificación más significativas son su origen español, el sistema de fortificaciones del Imperio Hispano, así como la colonización alemana del siglo XIX, que dejó rasgos notables en el diseño urbano y en la identidad colectiva actual de la ciudad. La valoración de este conjunto histórico constituye un caso de estudio interesante, tanto por los desafíos metodológicos que presenta, debido al carácter no acotado y disperso de los bienes objeto de estudio, como por las posibilidades de empleo de los resultados en planes de viabilidad de proyectos turísticos, o de evaluación de políticas de recuperación del patrimonio cultural.

Con respecto a los resultados de la evaluación de la disposición a pagar para los métodos de estimación, se observó que, para la muestra de los turistas, para acceder al circuito turístico preferido, la DAP fue de 8.327 pesos/ticket, en cambio para el caso de los ciudadanos de Valdivia, la disposición a contribuir a un fondo para el rescate y protección del patrimonio histórico fue de 9.028 pesos/anuales.

La factibilidad de la implementación de esta Fundación se presentó claramente rentable, al utilizar la tasa de descuento del 10\%, fue de 8,939 millones. La tasa interna de retorno, por su parte, alcanzó al 11,6\% lo cual nos indica que podemos usar una tasa social inferior a esta cantidad y el proyecto seguirá siendo rentable. Finalmente no debemos olvidar que en este tipo de proyecto deben tomarse en cuenta las externalidades que no son posibles de evaluar, tales como las relacionadas con el aumento de la oferta turística cultural en cantidad y calidad, repercusiones en comercio del sector como el de hotelería o restauración o en las mismas actividades culturales que pueda desarrollar la Fundación. Estas externalidades no han sido incluidas en la investigación por no contar con referentes mensurables ciertos y objetivos, pero ello redunda en el carácter cauteloso de los resultados aportados.

La formulación de este proyecto persigue, del mismo modo, la validación de una propuesta metodológica para la evaluación y el análisis de viabilidad de un plan de recuperación urbana y cultural en el caso de un país en vías de desarrollo, cuestión que resulta de enorme interés ante la escasez de experiencias relacionadas en este sentido, así como por la urgencia en la recuperación de muchos de estos elementos y la oportunidad de convertirlos en factores de desarrollo económico, o al menos de atracción turística. 


\section{BIBLIOGRAFÍA}

An, M.Y. y Ayala, R.A. (1996): “A simple Algorithm for Nonparametric Estimation of Distribution Functions with Arbitrarily Grouped Data”, Working Paper 9602, Department of Economics, Duke University, Durham.

Báez, A. (2007): “Aplicación de Técnicas de Valoración Contingente para la Evaluación del Patrimonio Histórico Urbano. El caso del Conjunto Histórico de la Ciudad de Valdivia (Chile)”, Tesis Doctoral, Departamento de Economía Aplicada, Universidad de Valladolid, Valladolid.

Banco Central de Chile. (2009): Base de datos económicos. www.bcentral.cl.

Bateman, I.J. y Willis, K.G. (1999): "Valuing Environmental Preferences. Theory and Practice of the Contingent Valuation in the US, EU and Developing Countrie". Oxford University Press, New York.

Bedate, A., Herrero, L.C. \& Sanz, A. (2006): "Ex Ante and Ex Post Valuations of a Cultural Good: The Case of a Museum of Contemporary Art”. 14th International Conference of the ACEI, Viena. Austria.

Bedate, A., Herrero, L.C. \& Sanz, A. (2009): "Economic valuation of a contemporary art museum: correction of hypothetical bias using a certainty question", Journal of Cultural Economics. Vol 27, nº 1. pp.159-176.

Bishop, R.C. y Heberlein, T.A. (1979): "Measuring Values of Extra-Market Goods: Are Indirect Measures Biased?”. American Journal of Agricultural Economics. pp.926-930.

Boardman, A., Greenberg, D., Vining, A., y Weimer, D. (2001): Cost-Benefit Analysis. Concepts and Practic. Segunda Edición, Prentice Hall, New Jersey.

Consejo de Monumentos Nacionales. (2008): Monumentos Nacionales de la Región de los Ríos. www.monumentos.cl.

Cameron, T.A. y James, M.D. (1987): "Efficient Estimation Methods for Use with "Closed-Ended "Contingent Valuation Survey Data". Review of Economics and Statistcs, n'69, pp. 269-276.

Cameron, T.A. y Quiggin J. (1994): "Estimation using contingent valuation data from a dichotomous choice with follow-up Questionnaire”. Journal of Environmental Economics and Management, n²7, pp. 218-234.

Carson, R.T., Mitchell, R.C., Conaway, M.C., Navrud, S. (1997): "Non-Moroccan Values for Rehabilitating the Fés Medina”, World Bank Report, Washington.

CEPAL. (2005): Metodología general de identificación, preparación y evaluación de proyectos de inversión pública. Instituto Latinoamericano y del Caribe de Planificación Económica y Social, Área de Proyectos y Programación de Inversiones. Santiago de Chile.

Chafla, P.R. (2001): Valor económico del patrimonio histórico arquitectónico del centro histórico de la ciudad de Quito. Tesis Doctoral, Departamento de 
Fundamentos de Economía e Historia Económica. Universidad de Alcalá. Madrid.

Comisión Europea (1997): Guide of costs-benefit analysis or investment projects. Evaluation Unit DG Regional Policy, European Commission.

Comisión Europea (2003): Guía del análisis costes-beneficios de los proyectos de inversión, Unidad de Evaluación DG Política Regional.

Cuccia, T. (2003): "Contingent Valuation" in TOWSE, R. (ed.) (2003). A Handbook of cultural economics, Chapter 14, Cheltenham: Edward Elgar Publishing, pp.119-131.

Cuccia, T. y Signorello, G. (2002): “A Contingent Valuation Study of willingness to Pay for Heritage Visits: Case Study of Noto". in I. RIZZO y R. TOWSE (eds.), The Economics of Heritage: a Study in the Political Economy of Culture in Sicily, Capítulo 10, Edward Elgar Publishing, Cheltenham, pp.147-163.

Greene, W. (1998): Análisis econométrico. Tercera Edición, Prentice Hall. Madrid.

Guarda, G. (1990): Flandes Indiano. Las fortificaciones del Reino de Chile 1541-1826, Ediciones Universidad Católica de Chile, Santiago.

Guarda, G. (2001): Nueva Historia de Valdivia. Ediciones Universidad Católica de Chile, Santiago.

Hanemann, W.M. (1984): "Welfare Evaluations in Contingent Valuation Experiments with Discrete Responses". American Journal of Agricultural Economic. n66, pp. 332-341.

Hanemann, W., Loomis, J. y Kaninnen, B. (1991): Statistical Efficiency of DoubleBounded Dichotomous Choice Contingent Valuation, American Journal of Agricultural Economics, $\mathrm{n}^{\circ} 73$, pp.1255-1263.

Harrison, G. W. (2006): "Experimental evidence on alternative environmental valuation methods". Environmental and Resource Economics, vol 34 n ${ }^{\circ}$, pp. 125162.

Harrison, G. W., y Rutström, E. E. (2005): "Experimental evidence on the existence of hypothetical bias in value elicitation methods". In C. R. Plott \& V. L. Smith (Eds.), Handbook of experimental economics results. Amsterdam: North-Holland.

Herrero, L.C. (2001): "Economía del patrimonio histórico”. Información Comercial Española. n ${ }^{\circ} 792$, pp.15-168.

Hutter, M. y Rizzo, I. (eds.), (1997): Economics Perspectives on Cultural Heritage. MacMillan Press Ltd., Basingstoke.

INE. (2009): Índice de Precios al Consumidor (IPC). www.ine.cl.

Mishan, E.J. (1971): Cost-Benefit Analysis.George Allen and Unwin. London.

Mitchell R.C. y Carson R. (1989): Using Surveys to Value Public Goods: The Contingent Valuation Method, Resources for the Future. Washington.

National Oceanographic Atmospheric Administration. (1994): Proposed rules for valuing environmental damages. Federal Register, vol 59, nº5, pp.1062- 
1191

Navrud, S. y Ready, R.C. (eds.) (2002): Valuing Cultural Heritage: Applying Environmental Valuation Techniques to Historic Buildings, Monuments and Artifacts. Edward Elgar Publishing, Cheltenham.

ONUDI. (1993): Guidelines for project evaluation. in www.unido.org.

Pagiola, S. (1999): Valuing the Benefits of Investments in Cultural Heritage: The Historic Core of Split. Environmental Department, World Bank. Washington, D.C.

Peacock, A. (ed.). (1998): Does the Past have a Future?. The Political Economy of Heritage. Institute of Economic Affairs, London.

Pearce, D. y Nash, C. (1981): The social appraisal of Projects: A text in Cost - Benefit Analysis. MacMillan, London.

Rus del G. (2004): Análisis Coste-Beneficio. Evaluación económica de políticas y proyectos de inversión, Ariel, Barcelona.

Santagata, W. y Signorello, G. (2000): "Contingent valuation of a cultural public good and policy design: The Case Napoli Musei Aperti”. Journal of Cultural Economics. n²4, pp.181-204.

Sanz J.A., Herrero, L.C. y Bedate, A. (2003): Contingent Valuation and Semiparametric Methods: A Case Study of the National Museum of Sculpture in Valladolid, Spain. Journal of Cultural Economics, vol 27, n³-4, pp. 241-257.

Saz, S. Del y Montagud, J. (2005): "Valuing cultural heritage: the social benefits of Restoring an old Arab Tower”. Journal of Cultural Heritage. n ${ }^{\circ} 6$, pp. 6977.

Sen, A. (2000): "The Discipline of Cost-Benefit Analysis". The Journal of Legal Studies, vol. 29, n², pp. 931-952.

Throsby, D. (2001): “Cultural Capital”. Journal of Cultural Economics, nº23, pp. 3-12.

Tuan, T.H. y Navrud, S. (2007): "Valuing cultural heritage in developing countries: comparing and pooling contingent valuation and choice modelling estimates". Environmental and Resource Economics, vol 38, n⿳⺈, pp. 5169.

Recibido: 29/10/2010

Aceptado: 04/06/2012

Arbitrado anónimamente 